\title{
Compact Wide-Band and Low Mutual Coupling MIMO Metamaterial Antenna using CPW Feeding for LTE/Wimax Applications
}

\author{
Duong Thi Thanh $\mathrm{Tu}^{1,2}$, Nguyen Tuan $\mathrm{Ngoc}^{1}$, Vu Van Yem ${ }^{2}$ \\ ${ }^{1}$ Faculty of Telecommunications 1, Posts and Telecommunications Institute of Technology, Hanoi, Vietnam \\ ${ }^{2}$ School of Electronics and Telecommunications, Hanoi University of Science and Technology, Hanoi, Vietnam \\ Correspondence: Duong Thi Thanh Tu, tudtt@ptit.edu.vn \\ Communication: received 12 May 2016, revised 23 January 2017, accepted 8 May 2017 \\ Online early access: 8 November 2018, Digital Object Identifier: 10.32913/rd-ict.vol2.no15.676 \\ The Area Editor coordinating the review of this article and deciding to accept it was Assoc. Prof. Nguyen Van Duc
}

\begin{abstract}
In this paper, a metamaterial antenna is designed by using coplanar waveguide (CPW) feeding to obtain wideband and compact size. The Multiple-Input Multiple-Output (MIMO) antenna is constructed by placing side-by-side two single metamaterial antennas which are based on the modified composite right/left handed (CRLH) model. The proposed antenna covers $22 \%$ of the experimental bandwidth for both cases of single and MIMO antennas. Implemented in FR4 substrate with the height of $1.6 \mathrm{~mm}$, the antenna is compact in size with radiating patch dimension of $5.75 \times 14 \mathbf{m m}^{2}$ at $3.5 \mathrm{GHz}$ resonant frequency that is suitable for Long Term Evolution (LTE)/Wimax applications in handheld devices. Furthermore, the combination of Defected Ground Structure (DGS) and enlarged ground of coplanar structure has solved the challenge of mutual coupling between elements in the MIMO metamaterial antenna using CPW feeding. With the distance of $0.46 \lambda_{0}$ between feeding points, the MIMO antenna obtains the high isolation of under $-20 \mathrm{~dB}$ for a huge bandwidth with a good agreement between simulations and measurements.
\end{abstract}

Keywords: Multiple-input multiple-output (MIMO), metamaterial antenna, coplanar waveguide $(C P W)$, low mutual coupling, defected ground structure (DGS).

\section{INTRODUCTION}

To satisfy high demands of users such as high data transfer rate and fast access, wireless communication technologies have been developed rapidly in recent years. Among them is the MIMO technology which has been deployed in terminals of modern wireless communication systems such as: $802.11 \mathrm{n}, 802.11 \mathrm{ac}, 802.11 \mathrm{ad}, 802.16 \mathrm{~m}$, LTE, LTEadvanced, and $5 \mathrm{G}$ systems. The most significant feature of MIMO is a high increase in channel capacity without bandwidth addition or increase of transmission power [1]. However, MIMO antenna systems require high isolation between antenna elements and compact size to be applied for portable devices [2].

There are several techniques used to decrease the size of antennas, such as incorporating a shorting pin in a microstrip patch [3], using short-circuit [4], or cutting slots in radiating patch with the fractal configuration [5]. Although these methods have achieved quite impressive compact size, they face challenges in terms of efficiency and gain reduction. On the other hand, using metamaterial is another method that provides an opportunity for designing small-dimension antennas with low cost and better performance parameters at both antenna and system levels [6, 7]. Besides, by using coplanar waveguide feeding, the metamaterial antenna is able to enlarge bandwidth [8].

Furthermore, coupling between microstrip antennas is important in a MIMO system. Mutual coupling between antenna elements is an unwanted phenomenon that distorts the behavior of the radiating elements. In MIMO systems, each antenna affects other elements that are closely packed by radiating over the air or by propagating surface currents through the ground plane. Thus, the performance of antennas tends to drop, especially for MIMO metamaterial antennas. Many methods have been proposed to decrease mutual coupling between antenna elements, such as grooving dielectric, covering the patch by dielectric layers, or using shorting pins to cancel the capacitive polarization currents of the substrate. However, one technique widely used in antenna designs recently is using metamaterial structures such as DGS and Electromagnetic Band Gap 


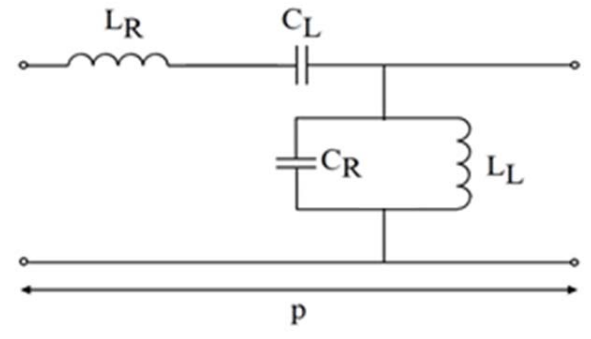

Figure 1. Equivalent circuit model of a CRLH-TL unit cell.

(EBG). These structures use the dielectric making a band gap structure between antenna elements, hence the isolation between them is improved significantly. Despite this, researchers prefer DGS techniques to EBG for suppressing element mutual coupling because they produce the band rejection characteristics similar to EBG structures but with more compact size [9-11].

There are several research works about mutual coupling reduction of metamaterial MIMO antennas. The MIMO antennas in [11-13] obtain high isolation between antenna elements but distances between feeding points are rather long. In [14], the MIMO metamaterial antenna achieves compact size and low mutual coupling of $-45 \mathrm{~dB}$ but the bandwidth has decreased significantly from $14.67 \%$ for a single antenna down to $6.25 \%$ for MIMO. This is a challenge in designing MIMO metamaterial antennas using CPW feeding.

In this paper, a wide-band MIMO metamaterial antenna using CPW feeding is proposed. Based on the FR4 substrate with the height of $1.6 \mathrm{~mm}$, the antenna obtains compact radiating elements with a patch size of $5.75 \times 14 \mathrm{~mm}^{2}$ and operates at the frequency band of 3.1-3.8 GHz. Using the combination of DGS and enlarged ground of coplanar structure, the MIMO antenna achieves low mutual coupling with a distance of $0.46 \lambda$ while covering $22 \%$ of the experimental bandwidth for both cases of single and MIMO antennas.

\section{Theory AND Design OF Metamaterial ANTENNA}

An equivalent circuit model of the fundamental composite right/left-handed transmission line (CRLH-TL) unit cell is presented in Figure 1. From this figure, the series capacitance $\left(C_{L}\right)$ and the shunt inductance $\left(L_{L}\right)$ as well as the series inductance $\left(L_{R}\right)$ and the shunt capacitance $\left(C_{R}\right)$ are determined in [6].

The resonant frequency of the antenna is calculated from the equivalent circuit. By using Bloch-Floquet theorem for a

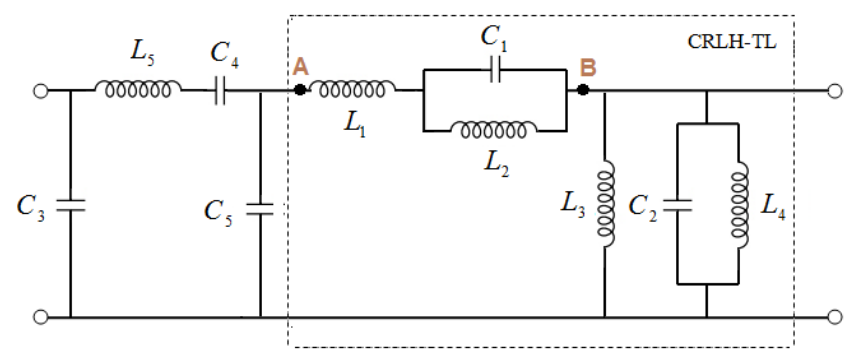

Figure 2. Equivalent circuit of the proposed metamaterial antenna.

CRLH-TL unit cell with periodic structures, the dispersion relation of the line is determined by the following formulas:

$$
\beta(w)=\frac{1}{p} \cos ^{-1}\left[1-\frac{1}{2}\left(\frac{w^{2}}{w_{R}^{2}}+\frac{w_{L}^{2}}{w^{2}}-\frac{w_{L}^{2}}{w_{s e}^{2}}-\frac{w_{L}^{2}}{w_{s h}^{2}}\right)\right],
$$

where $\beta$ is the propagation constant of Bloch wave, $p$ is the physical length of the unit cell, and $w$ is an angular velocity of the metamaterial antenna which is a variable of $\beta$ function, $\beta(w)$,

$$
\begin{aligned}
& w_{R}=\frac{1}{\sqrt{L_{R} C_{R}}}, \\
& w_{L}=\frac{1}{\sqrt{L_{L} C_{L}}}, \\
& w_{s e}=\frac{1}{\sqrt{L_{R} C_{L}}}, \\
& w_{s h}=\frac{1}{\sqrt{L_{L} C_{R}}} .
\end{aligned}
$$

The resonant condition of the CRLH-TL is obtained by the following condition [7]:

$$
\beta_{n} p=\frac{n \pi p}{l}=\frac{n \pi}{N},(n=0, \pm 1, \pm 2, \ldots, \pm(N-1)),
$$

where $n, N, l$ are the resonant mode, number of unit cell, and the total length of the resonator, respectively. When $n=0$, the wavelength becomes infinite and the resonant frequency of zero mode (ZOR) becomes independent of the antenna size, hence it can make the antenna compact.

\section{Single Metamaterial Antenna}

The structure of the proposed antenna is illustrated in Figure 3 with the equivalent circuit shown in Figure 2. The antenna is based on CRLH-TL and CPW feeding for reducing the size and achieving wide band.

In this design, the inductance $L_{2}$ and the capacitor $C_{1}$ are realized by a larger part of meander line while $C_{2}$ and $L_{4}$ are formed in a similar way by a smaller one. The inductance $L_{3}$ is realized by the line connecting the meander line to the ground. The radiation patch shapes the inductance $L_{1}$. The capacitors $C_{3}, C_{4}$, and $C_{5}$ are formed between two strips 


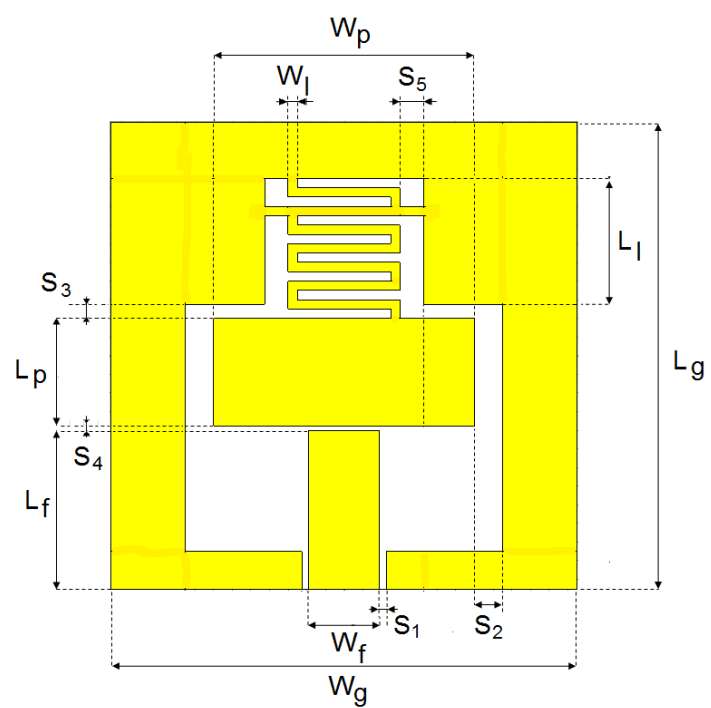

Figure 3. Configuration of the proposed CRLH-TL antenna.

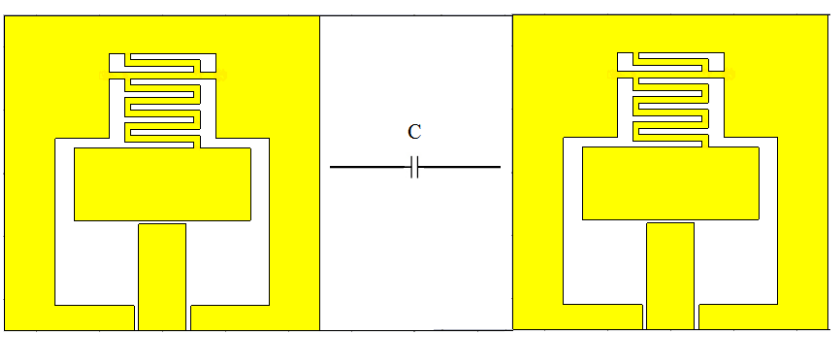

Figure 4. Configuration of the metamaterial MIMO antenna.

separated by distances of $S_{1}, S_{4}$, and $S_{2}$, respectively. The impedance of the $\mathrm{AB}$ circuit is obtained by the following equation:

$$
\begin{aligned}
Z_{A B} & =w L_{1}+\frac{w L_{2}}{1+w^{2} L_{2} C_{1}} \\
& =w L_{1}+\frac{1}{\frac{1}{w L_{2}}+w C_{1}} \\
& =w L_{1}+\frac{1}{w\left(\frac{1}{w^{2} L_{2}}+C_{1}\right)} \\
& =w L_{R}+\frac{1}{w C_{L}} .
\end{aligned}
$$

Thus, the series and shunt inductances $L_{R}$ and $L_{L}$, respectively, as well as the capacitors $C_{R}$ and $C_{L}$ are calculated by the following equations:

$$
\begin{aligned}
L_{R} & =L_{1} \\
L_{L} & =\frac{L_{3} L_{4}}{L_{3}+L_{4}}, \\
C_{R} & =C_{2} \\
C_{L} & =\frac{1}{w^{2} L_{2}}+C_{1} .
\end{aligned}
$$

TABLE I

Geometric Dimension of the Proposed Single Antenna

\begin{tabular}{|c|c|c|c|}
\hline Parameter & Value $(\mathrm{mm})$ & Parameter & Value $(\mathrm{mm})$ \\
\hline$L_{g}$ & 25 & $L_{1}$ & 6.75 \\
\hline$W_{p}$ & 14 & $S_{1}$ & 0.35 \\
\hline$L_{p}$ & 5.75 & $S_{2}$ & 1.5 \\
\hline$W_{f}$ & 3.8 & $S_{3}$ & 0.75 \\
\hline$L_{f}$ & 8.5 & $S_{4}$ & 0.25 \\
\hline$W_{1}$ & 0.5 & $S_{5}$ & 1.25 \\
\hline
\end{tabular}

For operating at $3.5 \mathrm{GHz}$, all detailed dimensions of the antenna are calculated and optimized by the CST (Computer Simulation Technology) software and shown in Table I. Using the FR4 substrate with the height of $1.6 \mathrm{~mm}$, dielectric constant of 4.4 and loss tangent of 0.02 , the antenna has compact radiation patch size of $5.75 \times 14 \mathrm{~mm}^{2}$ which is quite suitable for LTE-A tablets or Wimax applications.

\section{MIMO Antenna}

In the classical array design, the distance between the elements has been kept to around $0.5 \lambda$ to minimize unwanted coupling between the elements of an array and to minimize the spatial correlation. Here, we optimize this distance using simulation results from $0.4 \lambda$ to $0.5 \lambda$ to estimate its effect to $S$ parameters. Thus, our metamaterial MIMO model is constructed by placing two single antennas side by side at the distance of $0.46 \lambda_{0}$ from feeding point to feeding point.

The layout of the antenna is shown in Figure 4 with the total antenna size of $25 \times 65 \mathrm{~mm}^{2}$. The performance of the MIMO antenna using coplanar significantly decreases because of the capacitor $C$ which is formed between two strips separated by a distance of the antenna from edge to edge. To solve this problem, a combination of etching a novel DGS structure and enlarging the ground of the antenna on the surface plane is proposed as illustrated in Figure 5.

For decreasing the mutual coupling at $3.5 \mathrm{GHz}$ operating frequency over a large band, detailed dimensions of the MIMO antenna with the DGS structure as well as enlarged ground on the surface are optimized by CST software and shown in Table II.

\section{Simulation Results}

\section{Single Antenna}

Simulation of the prototype antenna using CST software is presented in this part. Current distributions of the meta- 


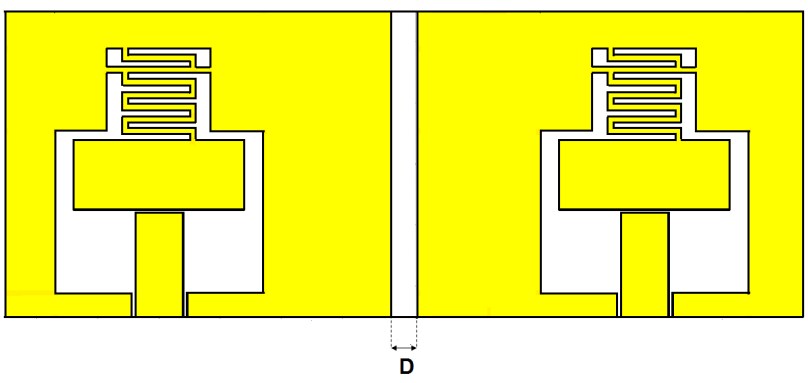

(a) Top plane

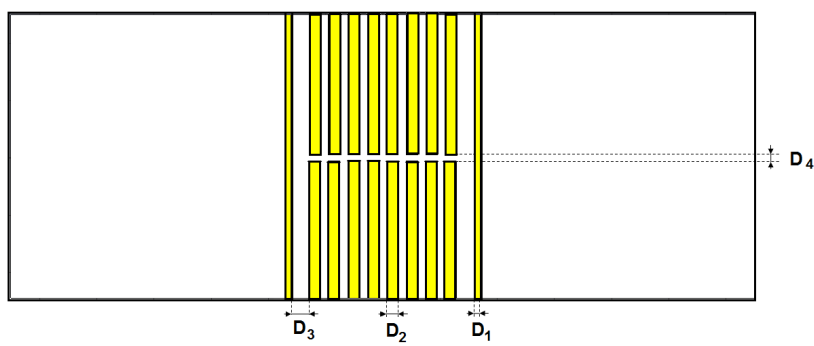

(b) Bottom plane

Figure 5. Configuration of the proposed metamaterial MIMO antenna with etching DGS structure and enlarging ground on the surface.

TABLE II

The Geometric Dimensions of the MIMO AnTEnNA

\begin{tabular}{|c|c|}
\hline Parameter & Value $(\mathrm{mm})$ \\
\hline$D_{1}$ & 0.5 \\
\hline$D_{2}$ & 1 \\
\hline$D_{3}$ & 1.5 \\
\hline$D_{4}$ & 0.5 \\
\hline$D$ & 2 \\
\hline
\end{tabular}

material antenna at the center frequency of LTE/ Wimax application are exhibited in Figure 6. As observed in this figure, the current distribution on the antenna at $3.5 \mathrm{GHz}$ mainly focuses on the meander strips instead of radiating patch as the principle of microstrip antennas. Thus, the resonant frequency of the proposed metamaterial antenna depends on the dimensions which are contributed by the CRLH structure such as $S_{3}$ and $S_{5}$.

Figure 7 illustrates how the $S_{11}$ of the single metamaterial antenna varies with different values of $S_{3}$ and $S_{5}$. To obtain the resonant frequency of $3.5 \mathrm{GHz}$, all dimensions of the proposed metamaterial antenna is optimized by CST software. The $S$ parameter of the single metamaterial antenna is shown in Figure 8. It is obvious that the antenna operates at $3.5 \mathrm{GHz}$ with a large bandwidth of $1.03 \mathrm{GHz}$ (29.4\%). The reflection coefficient is $-40 \mathrm{~dB}$ at the resonant frequency.

The 2D and 3D radiation patterns of the proposed antenna are illustrated in Figure 9 that is suitable for handheld devices with dipolar and smooth radiation. Besides, the

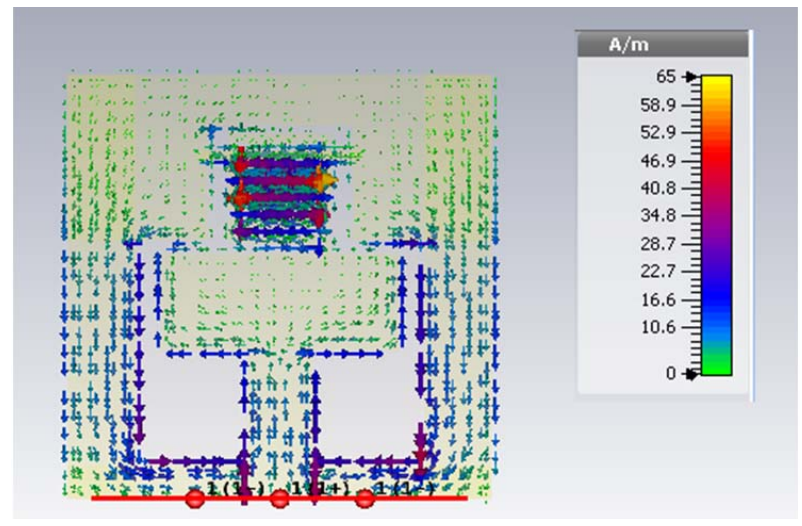

Figure 6. Surface current distribution on the single antenna.

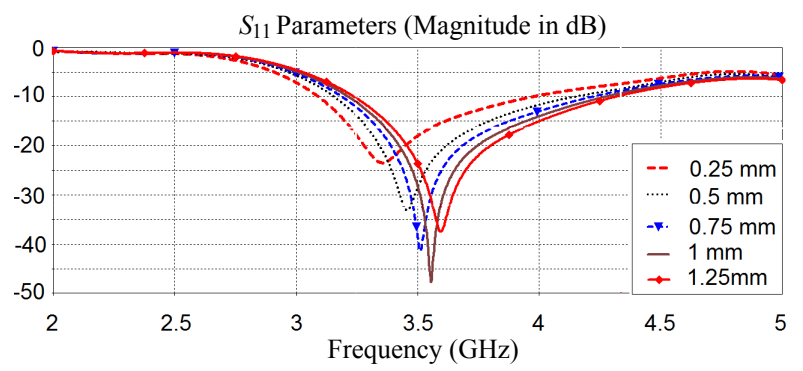

(a)

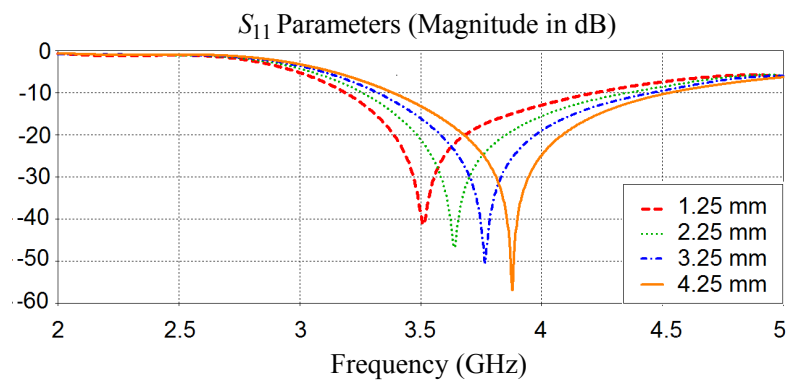

(b)

Figure 7. $S_{11}$ results of the single metamaterial antenna for different values of (a) $S_{3}$ and (b) $S_{5}$.

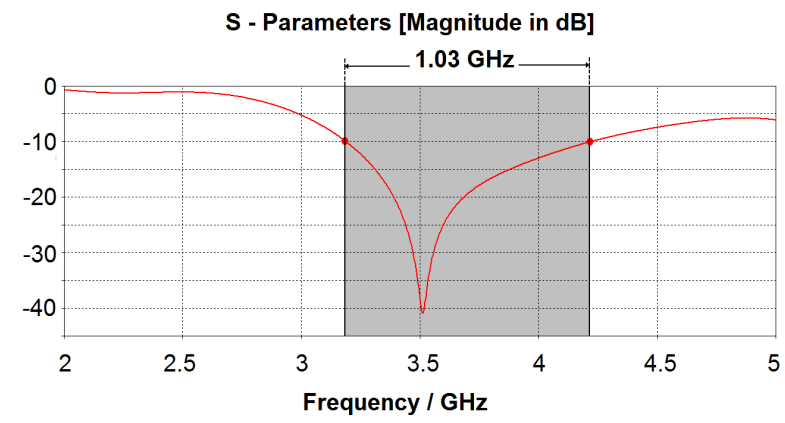

Figure 8. $S_{11}$ parameter of the single metamaterial antenna.

antenna radiation efficiency of $71 \%$ and gain of $1.96 \mathrm{~dB}$ are rather good at $3.5 \mathrm{G} \mathrm{Hz}$ resonant frequency with dipolar radiation pattern [20]. 
TABLE III

COMPARISON BETWEEN THE PROPOSED DESIGN AND PREVIOUS SINGLE META ANTENNAS

\begin{tabular}{|l|c|c|c|c|c|c|}
\hline & {$[15]$} & {$[16]$} & {$[17]$} & {$[18]$} & {$[19]$} & Our design \\
\hline Freq. $(\mathrm{GHz})$ & 5.55 & 1.94 & 2.1 & 2.14 & 8.45 & 3.5 \\
\hline Bandwidth (\%) & 4.3 & 10.3 & 15.1 & 7.84 & 22.9 & 29.4 \\
\hline Radiation Efficiency $(\%)$ & 75.6 & 85 & 72 & 81.7 & 74 & 71 \\
\hline$S_{11}(\mathrm{~dB})$ & -32 & -25 & -32 & -25 & -30 & -40 \\
\hline Total size $\left(\lambda^{2}\right)$ & $0.63 \times 0.74$ & $0.18 \times 0.32$ & $0.14 \times 0.22$ & $0.18 \times 0.15$ & $0.22 \times 0.43$ & $0.29 \times 0.29$ \\
\hline Gain $(\mathrm{dBi})$ & 6.7 & 2.3 & 1.62 & 1.69 & 1.83 & 1.96 \\
\hline
\end{tabular}

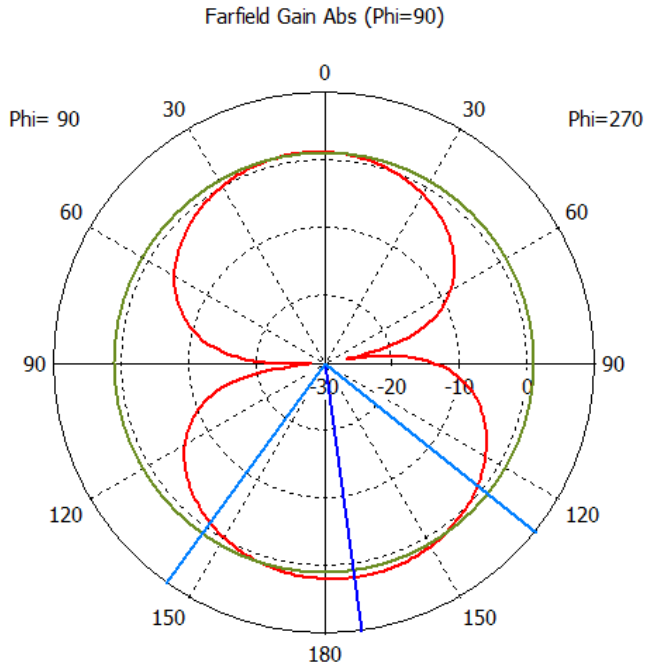

Theta / Degree vs. dB

(a) $2 \mathrm{D}$

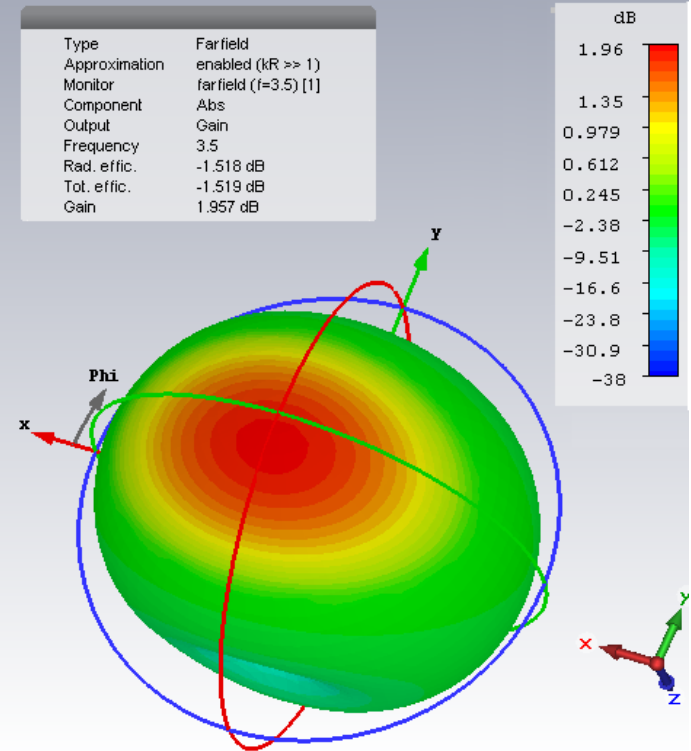

(b) $3 \mathrm{D}$

Figure 9. The radiation pattern of the single antenna.

In addition, Table III shows comparison between our proposed antenna and the previous meta antenna designs. It is clearly seen that, the resonant frequencies of all previous designs depend on ZOR and can not adjust to desired resonant frequency like our proposed antenna. Besides, the proposed antenna obtains much larger bandwidth than all previous designs.

\section{MIMO Antenna}

The simulation results of reflection coefficients of an initial MIMO antenna (without DGS and enlarge ground) are shown in Figure 10. From this figure, it is observed that the $S$ parameters of the initial MIMO antenna are changed and can not meet the isolation demand for MIMO antennas due to the effect of mutual coupling. In the operation band, the $S_{12}$ and $S_{21}$ are not below $-20 \mathrm{~dB}$.

This fact is clearly demonstrated by surface current distribution on the initial MIMO antenna shown in Figure 11. It is observed from this figure that when the first

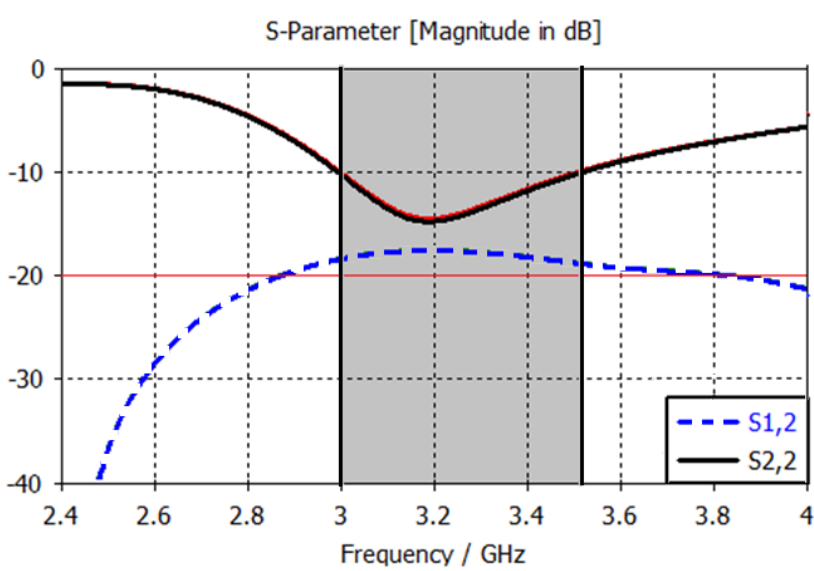

Figure 10. Simulated $S$ parameters of the initial MIMO antenna.

antenna element is excited from Port1, the surface current is strongly induced on the second antenna element at Port2, therefore the mutual couplings $\left(S_{12}\right.$ and $\left.S_{21}\right)$ are increased. 


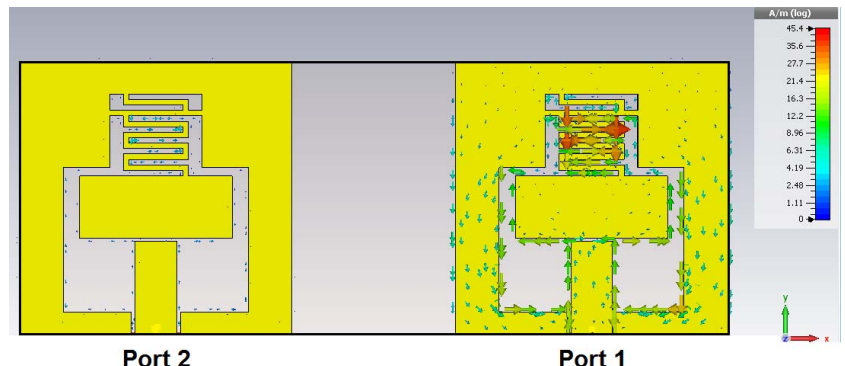

(a) Top plane

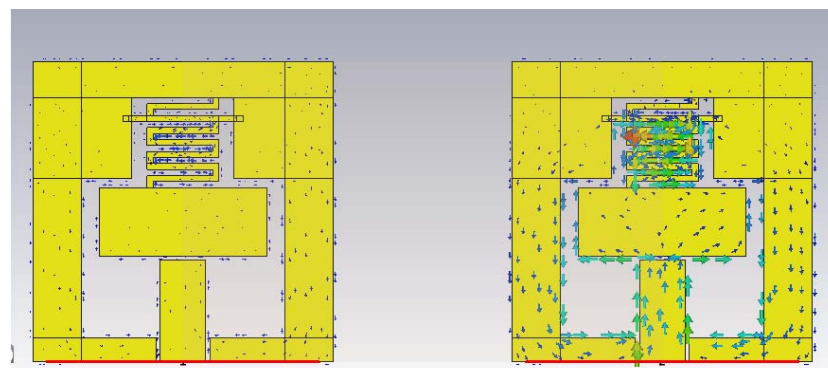

(b) Bottom plane

Figure 11. Surface current distribution at $3.5 \mathrm{GHz}$ on the initial metamaterial MIMO antenna.

To solve this challenge, a combination of DGS and enlarged ground structure is proposed. The enlarged ground leads the surface current affecting the DGS structure, hence the current distribution of the proposed MIMO antenna at $3.5 \mathrm{GHz}$ is focused on the DGS structure as shown in Figure 12. That is why the effect of the surface current from the first element to the second element is reduced as seen clearly in Figure 13. The $S_{12}$ is below $-20 \mathrm{~dB}$ all over the wide band. In addition, the combination of DGS and enlarged ground structure is able to improve the reflection coefficient. The reflection coefficient is decreased by $25 \mathrm{~dB}$ and obtains $-40 \mathrm{~dB}$ at the resonant frequency with an enlarged bandwidth compared to that of the initial MIMO antenna.

The $2 \mathrm{D}$ and $3 \mathrm{D}$ radiation patterns of the proposed MIMO model are shown in Figure 14. With low mutual coupling between antenna elements, the radiation pattern of the MIMO antenna is the same as of the single antenna. The gain is still good with dipolar radiation pattern.

In MIMO antenna systems, the correlation factor, which is also called the enveloped correlation coefficient (ECC), will be significantly degraded with higher coupling levels. This factor is calculated from radiation patterns or scattering parameters. For a simple two-port network, assuming uniform multipath environment, the enveloped correlation $\left(\rho_{e}\right)$ can be calculated conveniently and quickly from S-

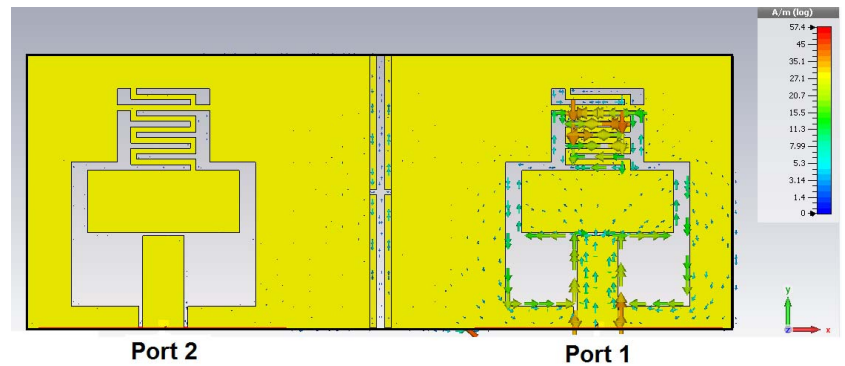

(a) Top plane

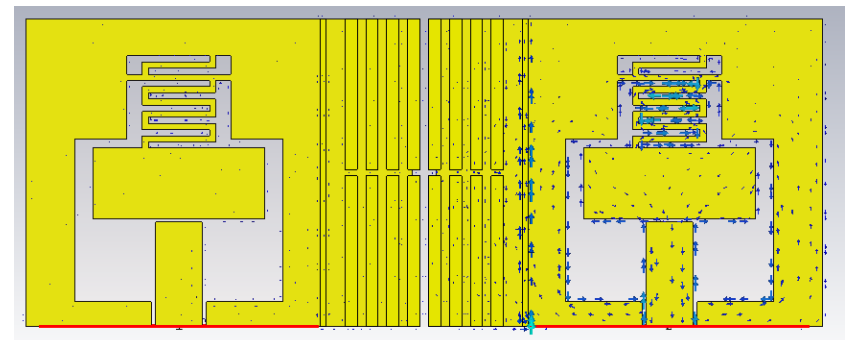

(b) Bottom plane

Figure 12. Surface current distribution at $3.5 \mathrm{GHz}$ on the proposed MIMO antenna with DGS and enlarged ground structure.

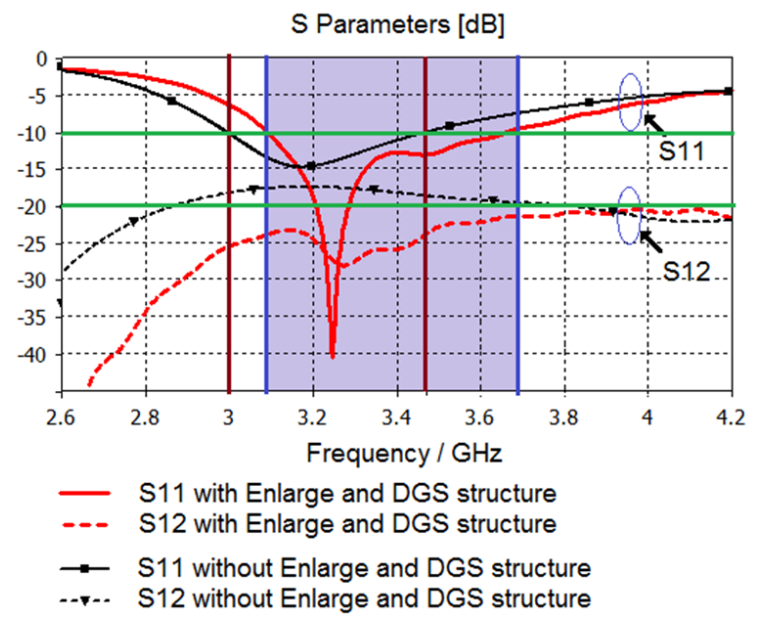

Figure 13. Simulated S parameters of the MIMO antenna.

parameters, as follows [21]:

$$
\rho_{e}=\frac{\left|S_{11}^{*} S_{12}+S_{21}^{*} S_{22}\right|^{2}}{\left(1-\left|S_{11}\right|^{2}-\left|S_{21}\right|^{2}\right)\left(1-\left|S_{22}\right|^{2}-\left|S_{12}\right|^{2}\right)}
$$

The correlation factor curve of the proposed MIMO antenna operating at $3.5 \mathrm{GHz}$ is shown in Figure 15. From this figure, the metamaterial MIMO antenna using combination of DGS and enlarged ground structure has simulated ECC lower than 0.004 for entire operational band. Therefore, it is quite suitable for mobile communications with a minimum acceptable correlation coefficient of 0.5 [22] as well as for LTE devices with $\rho_{e} \leq 0.3$ for the bands of interest [23]. 


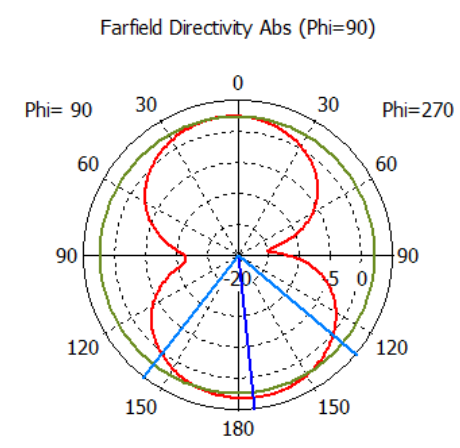

Theta / Degree vs. dBi (a) $2 \mathrm{D}$

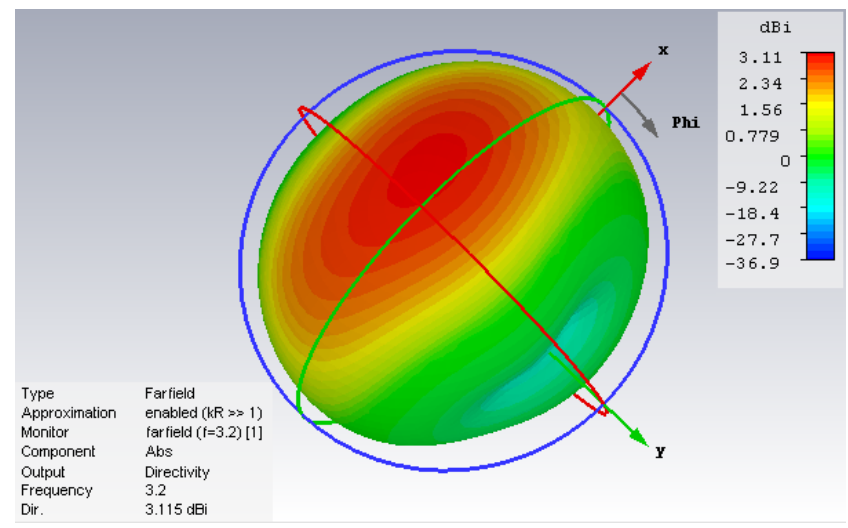

(b) $3 \mathrm{D}$

Figure 14. The radiation pattern of the proposed antenna.

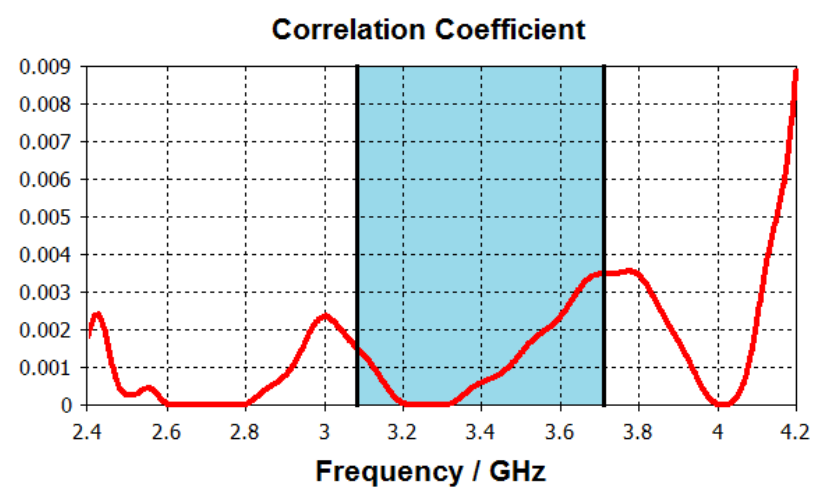

Figure 15. Correlation factor $\left|\rho_{e}\right|$ curve of the proposed MIMO antenna.

\section{Measurement Results}

To verify the performance of the proposed metamaterial antenna, the antennas are fabricated for both single and MIMO models on the FR4 substrate with the permittivity of 4.4 and the thickness of $1.6 \mathrm{~mm}$. Figure 16 shows a photo of the single antenna with an overall size of $25 \times 25 \times 1.6 \mathrm{~mm}^{2}$. The measurement result of the $S_{11}$ parameter is compared to the simulated result in Figure 17. It is clearly seen that the

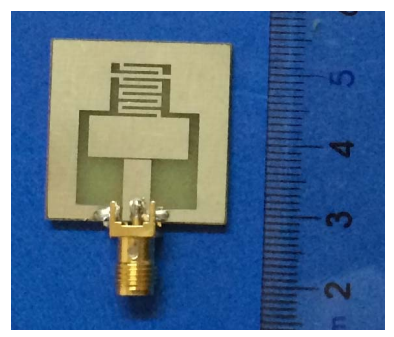

(a) Top plane

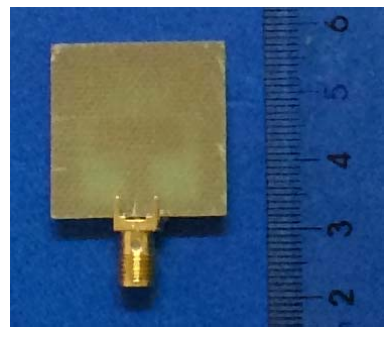

(b) Bottom plane
Figure 16. The fabricated single antenna.

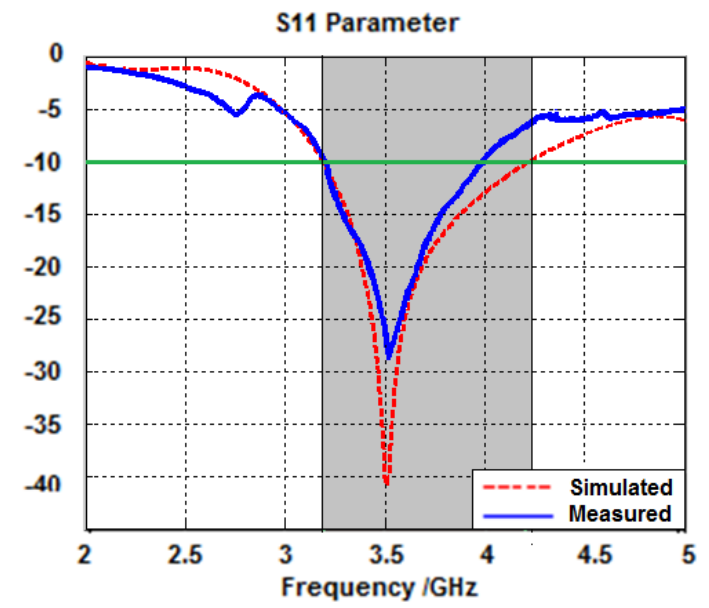

Figure 17. Comparison between measurement and simulated results of $S_{11}$ parameter of the single antenna.

single antenna operates at $3.5 \mathrm{GHz}$ with $22 \%$ bandwidth.

Figure 18 shows a photo of the MIMO antenna with an overall size of $25 \times 65 \times 1.6 \mathrm{~mm}^{2}$. The measurement and simulated results for the $S$-parameters are compared in Figure 19. It is observed that the MIMO antenna can also operate at $3.5 \mathrm{GHz}$ with $22 \%$ bandwidth.

From this measurement, it can be concluded that the measurement results agree well with the simulated ones. Moreover, using the combination of DGS structure and enlarged ground of coplanar structure, the MIMO antenna obtains low mutual coupling with the distance of $0.46 \lambda$ while ensuring $22 \%$ of the experimental bandwidth for both cases of single and MIMO antennas.

\section{Conclusion}

In this paper, novel metamaterial antennas using CPW feeding are proposed. Based on the CRLH-TL structure, the antennas obtain compact patch sizes of less than $30 \%$ compared to the conventional ones. Thanks to the combination of CRLH-TL structure and CPW feeding method, the proposed antenna obtains large experimental bandwidth of $22 \%$ for both cases of single and MIMO antennas. 


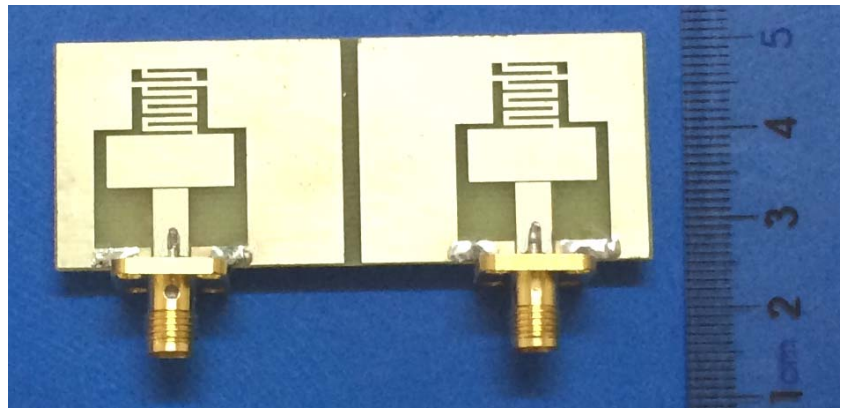

(a) Top plane

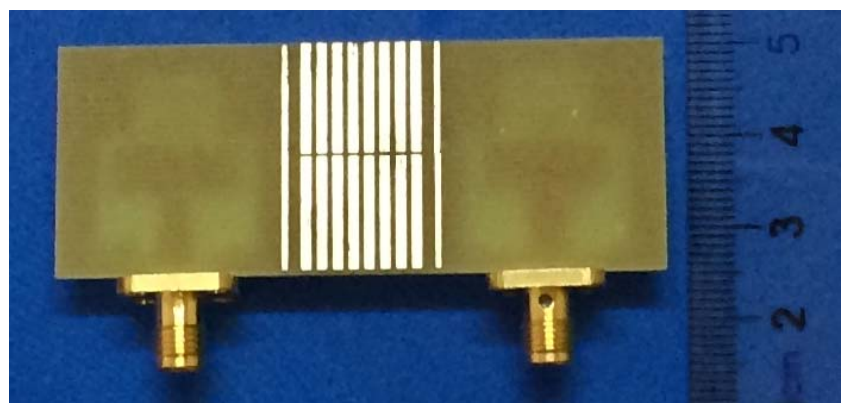

(b) Bottom plane

Figure 18. The fabricated MIMO metamaterial antenna using coplanar feeding.

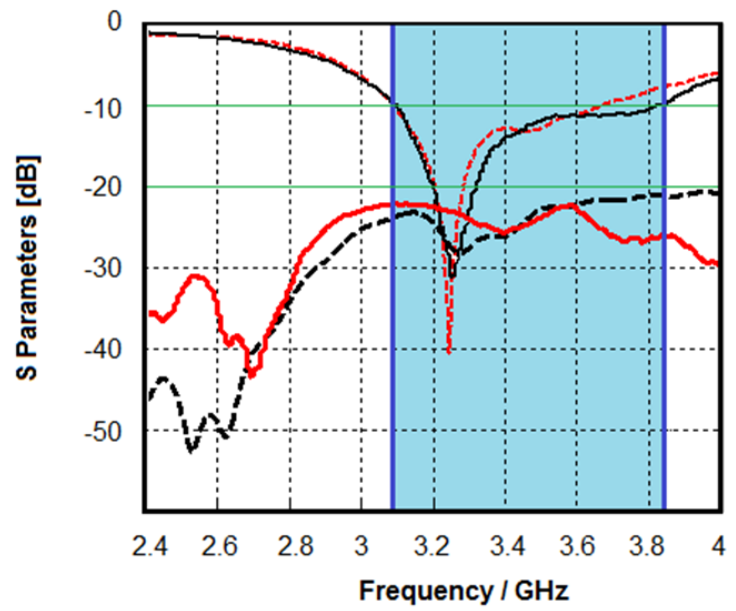

----- S11-Simulation _ $\quad$ S11-Measurement ---. S12-Simulation _ S12-Measurement

Figure 19. Comparison between measurement and simulated results of $S$-parameters of the proposed MIMO antenna.

Moreover, for reducing the mutual coupling between patch elements in the MIMO antenna, a combination of DGS structure and enlarged ground part on the surface plane is proposed. With high isolation for a large band of $600 \mathrm{MHz}$, the proposed structure of mutual coupling reduction is significant for wide-band commercial applications in modern wireless communications.

\section{ACKNOWLEDMENT}

This work is partly supported by Motorola Solutions Foundation under Motorola scholarship and research funding program for ICT education.

\section{REFERENCES}

[1] G. J. Foschini and M. J. Gans, "On limits of wireless communications in a fading environment when using multiple antennas," Wireless Personal Communications, vol. 6, no. 3, pp. 311-335, 1998.

[2] A. Jamil, M. Z. Yusoff, and N. Yahya, "Current issues and challenges of MIMO antenna designs," in Proceedings of the International Conference on Intelligent and Advanced Systems (ICIAS). IEEE, 2010, pp. 1-5.

[3] M.-C. Huynh and W. Stutzman, "Ground plane effects on planar inverted-f antenna (PIFA) performance," IEE Proceedings-Microwaves, Antennas and Propagation, vol. 150, no. 4, pp. 209-213, 2003.

[4] M. C. Scardelletti, G. E. Ponchak, S. Merritt, J. S. Minor, and C. A. Zorman, "Electrically small folded slot antenna utilizing capacitive loaded slot lines," in Proceedings of the Radio and Wireless Symposium. IEEE, 2008, pp. 731-734.

[5] F. Merli, L. Bolomey, J.-F. Zurcher, G. Corradini, E. Meurville, and A. K. Skrivervik, "Design, realization and measurements of a miniature antenna for implantable wireless communication systems," IEEE Transactions on Antennas and Propagation, vol. 59, no. 10, pp. 3544-3555, 2011.

[6] C. Caloz and T. Itoh, Electromagnetic metamaterials: transmission line theory and microwave applications. John Wiley \& Sons, 2005.

[7] N. Engheta and R. W. Ziolkowski, Metamaterials: physics and engineering explorations. John Wiley \& Sons, 2006.

[8] R. N. Simons, Coplanar Waveguide: circuits, components, and systems. Wiley, New York, 2001.

[9] S. Xiao, M.-C. Tang, Y.-Y. Bai, S. Gao, and B.-Z. Wang, "Mutual coupling suppression in microstrip array using defected ground structure," IET microwaves, antennas \& propagation, vol. 5, no. 12, pp. 1488-1494, 2011.

[10] F.-G. Zhu, J.-D. Xu, and Q. Xu, "Reduction of mutual coupling between closely-packed antenna elements using defected ground structure," Electronics Letters, vol. 45, no. 12, pp. 601-602, 2009.

[11] N. K. Kiem, H. N. B. Phuong, Q. N. Hieu, and D. N. Chien, "A novel metamaterial mimo antenna with high isolation for wlan applications," International Journal of Antennas and Propagation, vol. 2015, 2015.

[12] J. C. Rao and N. V. Rao, "Cpw-fed compact ultra wideband mimo antenna for portable devices," Indian Journal of Science and Technology, vol. 9, no. 17, pp. 1-9, 2016.

[13] M. A. Abdalla and A. A. Ibrahim, "Design of close, compact, and high isolation meta-material mimo antennas," in Proceedings of the Antennas and Propagation Society International Symposium (APSURSI). IEEE, 2013, pp. 186187.

[14] Y. Torabi, A. Bahri, and A.-R. Sharifi, "A novel metamaterial MIMO antenna with improved isolation and compact size based on LSRR resonator," IETE Journal of Research, vol. 62, no. 1, pp. 106-112, 2016.

[15] X. Li, Q. Feng, and Q.-Y. Xiang, "A novel via less resonant type antenna based on composite right/left-handed transmission line (crlh-tl) unit cell with defected ground structure," Progress In Electromagnetics Research, vol. 38, pp. 55-64, 2013. 
[16] P.-W. Chen and F.-C. Chen, "Asymmetric coplanar waveguide (ACPW) zeroth-order resonant (ZOR) antenna with high efficiency and bandwidth enhancement," IEEE Antennas and Wireless Propagation Letters, vol. 11, pp. 527-530, 2012.

[17] P.-L. Chi and Y.-S. Shih, "Compact and bandwidth-enhanced zeroth-order resonant antenna," IEEE Antennas and Wireless Propagation Letters, vol. 14, pp. 285-288, 2015.

[18] K. Sheeja, P. Sahu, and S. Behera, "Comparative study of a CRLH TL based Zeroth Order Resonant antenna," in Proceedings of the National Conference on Communications (NCC). IEEE, 2012, pp. 1-5.

[19] M. Tauseef Asim and M. Ahmed, "Metamaterial inspired microstrip antenna investigations using metascreens," International Journal of Antennas and Propagation, vol. 2015, 2015.

[20] ETSI TS 136101 V10.3.0 (2011-06), "Lte, user equipment (ue) radio transmission and reception," 2011.

[21] A. Lai, K. M. Leong, and T. Itoh, "Infinite wavelength resonant antennas with monopolar radiation pattern based on periodic structures," IEEE Transactions on Antennas and Propagation, vol. 55, no. 3, pp. 868-876, 2007.

[22] M. P. Karaboikis, V. C. Papamichael, G. F. Tsachtsiris, C. F. Soras, and V. T. Makios, "Integrating compact printed antennas onto small diversity/MIMO terminals," IEEE Transactions on Antennas and Propagation, vol. 56, no. 7, pp. 2067-2078, 2008.

[23] 3GPP TS 36.101, V8.3.0., "EUTRA User Equipment Radio Transmission and Reception," Sep. 2008.

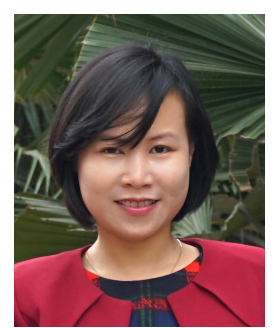

Duong Thi Thanh Tu received B.E, M.E degrees in Electronics and Telecommunications from Hanoi University of Science and Technology and National University in 1999 and 2005, respectively. She now is a lecturer at Faculty of Telecommunications 1, Posts and Telecommunications Institute of Technology. She presently is doing $\mathrm{PhD}$ at School of Electronics and Telecommunications, Hanoi University of Science and Technology. Her research interests include antenna design for next generation wireless networks as well as the special structure of material such as metamaterial, electromagnetic band gap structure.

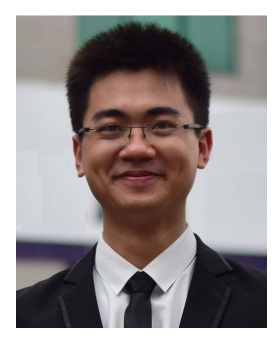

Nguyen Tuan Ngoc is a fifth-year student at Faculty of Telecommunications 1, Posts and Telecommunications Institute of Technology. Besides, he is also a part time collaborator at Viettel Network Technologies Center - VTTEK. His current research centers on antenna design for new generation wireless networks.

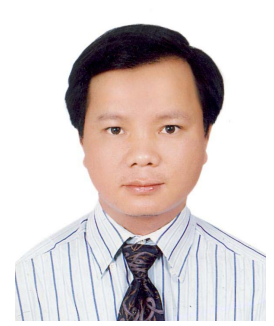

Vu Van Yem received $\mathrm{PhD}$ degree from the Department of Electronics and Communications, TELECOM ParisTech, France, in 2005. From 2006 to 2007, he was a postdoctoral researcher at the Department of Hyper-Frequency, the Institute of Electronics and Microelectronics and Nano technology (IEMN), France. He has been qualified as associate professor since 2009. He is the Head of the Department of Telecommunication Systems, School of Electronics and Telecommunications as well as Vice-Dean of Graduate School, Hanoi University of Science and Technology, Vietnam. His research interests are microwave engineering, antenna, chaos-based digital communications as well as advanced wireless communication and localization systems. 